Sādhanā Vol. 40, Part 4, June 2015, pp. 1105-1116. (C) Indian Academy of Sciences

\title{
Design of a CMOS PFD-CP module for a PLL
}

\author{
N K ANUSHKANNAN ${ }^{1, *}$ and H MANGALAM ${ }^{2}$ \\ ${ }^{1}$ Department of Electronics and Communication Engineering, Tamilnadu College \\ of Engineering, Coimbatore 641659, India \\ ${ }^{2}$ Department of Electronics and Communication Engineering, Sri Krishna College \\ of Engineering \& Technology, Coimbatore 641008, India \\ e-mail: anushkannan81@gmail.com; hmangalam2@gmail.com
}

MS received 3 September 2014; revised 23 January 2015; accepted 4 February 2015

\begin{abstract}
This paper introduces a modified design of Phase frequency detector (PFD) with reduced dead zone and improved charge pump (CP) with reduced current mismatch for a Phase Locked Loop (PLL). Three modified PFD circuits are proposed, designed, simulated, and the results are analyzed considering dead zone as a constraint. Design of pass transistor logic network plays a part in the diminution of the dead zone. Further, an improved design of CP is proposed to reduce current mismatch. It is achieved by placing the single ended differential amplifier in currentvoltage feedback configuration which offers high output impedance. Simulations are performed using T-SPICE, implemented in IBM $0.13 \mu \mathrm{m}$ technology under $1.3 \mathrm{~V}$ power supply. Results show that the modified PFD design has a dead zone of $0.3 \mathrm{~ns}$ and the current mismatch decrements to $0.1 \mu \mathrm{A}$ in an improved $\mathrm{CP}$ design.
\end{abstract}

Keywords. Dead zone; phase frequency detector; pass-transistor; charge pump; current mismatch.

\section{Introduction}

A Phase Locked Loop (PLL) is a closed loop feedback control system which is capable of generating a clock signal that has a fixed relationship to the reference clock signal. It causes a particular system to track with another one (Best 2003). PLL adjust their output signal about the reference signal either by lowering or elevating the frequency of Voltage Controlled Oscillator (VCO) until the output is checked to the reference signal in terms of both frequency and phase. The primary aim of a PLL is to get a signal in which the phase is the same as the phase of a reference signal (Elserougi et al 2006).

There will be continuous iterations of comparison of the reference and feedback signals. The phase of the reference and feedback signal is zero when PLL is in lock mode. Moreover, the PLL continues to compare the two signals, but since they are in lock mode, the PLL output is constant (NehaPathak \& Ravi Mohan 2014).

${ }^{*}$ For correspondence 


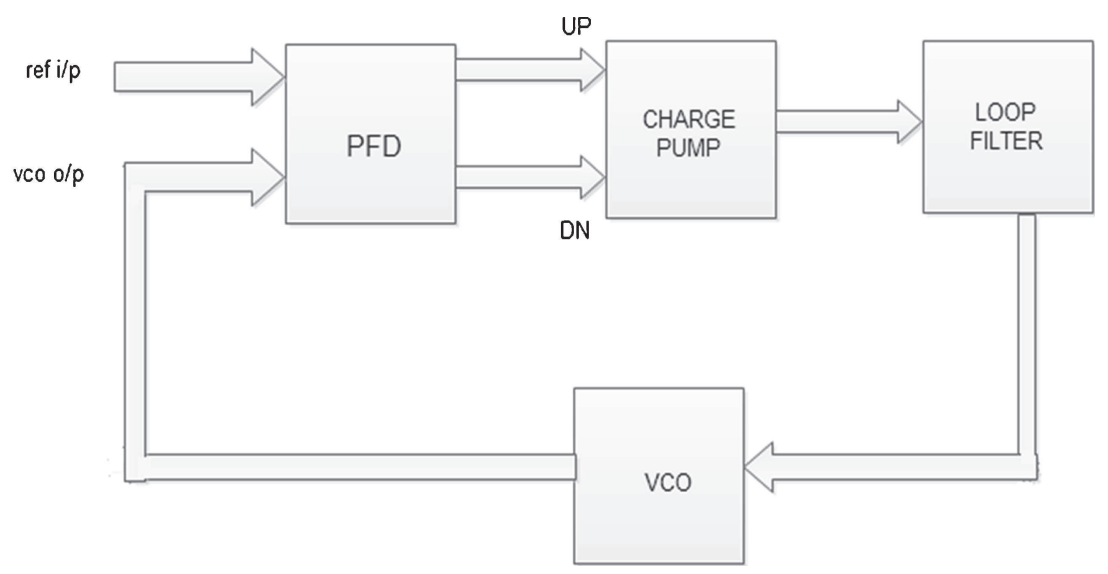

Figure 1. Block diagram of the phase locked loop.

Figure 1 represents the overall block diagram of PLL. It consists of a phase frequency detector (PFD), charge pump (CP), low pass filter (LP), and VCO (Lee et al 2003, 2010). The role of PFD is to compare the two input analog signals in terms of both phase and frequency and produce their difference as a digital output voltage (Al Sabbagh 2007). Since the output from PFD is digital, before feeding that signal to the filter, $\mathrm{CP}$ converts into analog voltage. In order to inhibit the unwanted high frequencies, the analog signal is given to low pass loop filter.

VCO is a voltage controlled oscillator in which the produced output signal frequency is dependent on the input voltage of the VCO. Thus, by adjusting the VCO output frequency, PLL is phase locked. The traditional PFD uses two D Flip Flop (FF) and NAND gate at its reset path (Nidagundi et al 2013). This module identifies the phase difference between ref input (i/p) and the vco output (o/p) and produces two output signals UP and DN (Wen-Ching Chang et al 2002).

Conventional PFD suffers from a major problem called dead zone. The dead zone is a situation where the PFD cannot detect the phase difference at the rising edge of the two clock signals so that it compares when close to each other. During this state PFD can lock to a wrong phase. This false detection is mainly due to circuit mismatch and delay mismatch. The traditional CP produces an error current proportional to the error signal from PFD. The CP comprises two current sources (Mano et al 2013). The purpose of current sources is to push the charge in and out of the filter. PFD produces the output UP signal which enables UP switch to turn on in CP. Then the CP injects the current into the loop filter. Similarly, the DN signal turns on the DN switch in the CP that causes sink current from the loop filter. The current over UP and DN switch must be equal to avoid current mismatch.

This paper reports new proposal of three modified circuits for PFD in order to reduce the dead zone and improved circuit in order to minimize the current mismatch in CP. The remainder of the paper is organized as follows, Section 2 summarizes a review of PFD and CP. Section 3 briefs the proposed circuits followed by the simulation results and conclusion in sections 4 and 5 , respectively.

\section{Literature survey}

There are various types of PFDs and CP circuits for designing PLL. Existing modules of PFD have pros and cons of their own. In addition to conventional PFD design, Pass transistor based 
PFD design with single edge clock produces output, but with more power consumption (Mansuri et al 2002). Dynamic D FF based PFD design uses two inverters in the precharged state to avoid short circuit in the device. However, the circuit requires additional delay elements to reduce the dead zone (Khare et al 2008).

Another possible approach is to delay the input rising edge instead of delaying the entire input pulse. It is termed as the Delayed-Input-Edge Dynamic PFD design (DIE-PFD) (Zhang \& Syrzycki 2010). All studied PFD modules are designed, simulated and observed. In order to achieve higher performance, Optimization such as transistors sizing is done to suppress switching delays in Pass Transistor D FF. From the Comparative analysis of the existing modules which are simulated, it is found that Pass transistor PFD design has maximum dead zone (AnushKannan et al 2013a, b).

The performance of CP depends heavily on the ability to efficiently generate high voltages on-chip while meeting stringent power and area requirements (AnushKannan et al 2013b). For CPs, various works have been carried out by researchers' in order to obtain the current signal matched with the signal from PFD. Error amplifier based CP (Lee et al 2000) circuit produces output, however no perfect current matching characteristics. High Gain Operational Amplifier (OP-AMP) based CP (Talwekar \& Limaye 2010) circuit which has a differential amplifier produces output but with glitches. Master Slave CP with two identical CP circuits (Bai et al 2014) produces output with current mismatch. Low jitter CP (Shi 2008) circuit produces output with the problem of charge sharing. In all different circuits current mismatch is the common problem which is taken as a constraint for minimization.

\section{Proposed design}

Although there are many existing designs, it is necessary to provide an absolute solution for the dead zone in PFD and the current mismatch problem in CP. Among all the PFD design architectures that already existed, it is found from the literature Survey that the Pass Transistor Based D flip flop PFD design has maximum dead zone. In order to reduce the dead zone, it is proposed to design three modified PFD circuits.

\subsection{Proposed pass transistor AND based PFD design}

Figure 2 illustrates the modified design of the Pass Transistor based PFD (Mansuri et al 2002). A pass transistor logic AND gate (Markovic et al 2000) is introduced by replacing the NAND gate in the existing circuit. In addition, there are two inverters in order to reduce dead zone. Modifications minimize clock skew and thereby results in dead zone reduction. The operations are similar to the original one except that the AND gate and two inverters are put back to the reset path. This design reduces the dead zone. The pass transistor logic design can reduce the complex structures into a simple structure which increases the operating speed (Whitaker 1983). Hence, this proposed design reduces the delay due to use of pass transistor logic and modifications in the reset path reduce power consumption and size.

\subsection{Proposed delayed input edge (DIE) pass transistor PFD design}

Figure 3 illustrates the modified circuit of DIE- Dynamic PFD (Zhang \& Syrzycki 2010). As the objective is to reduce the dead zone for pass transistor based PFD, DIE concept is used here. For this purpose, N-MOS pass transistor is applied at the input. Pass transistor based NAND gate acts as a controlling element. 


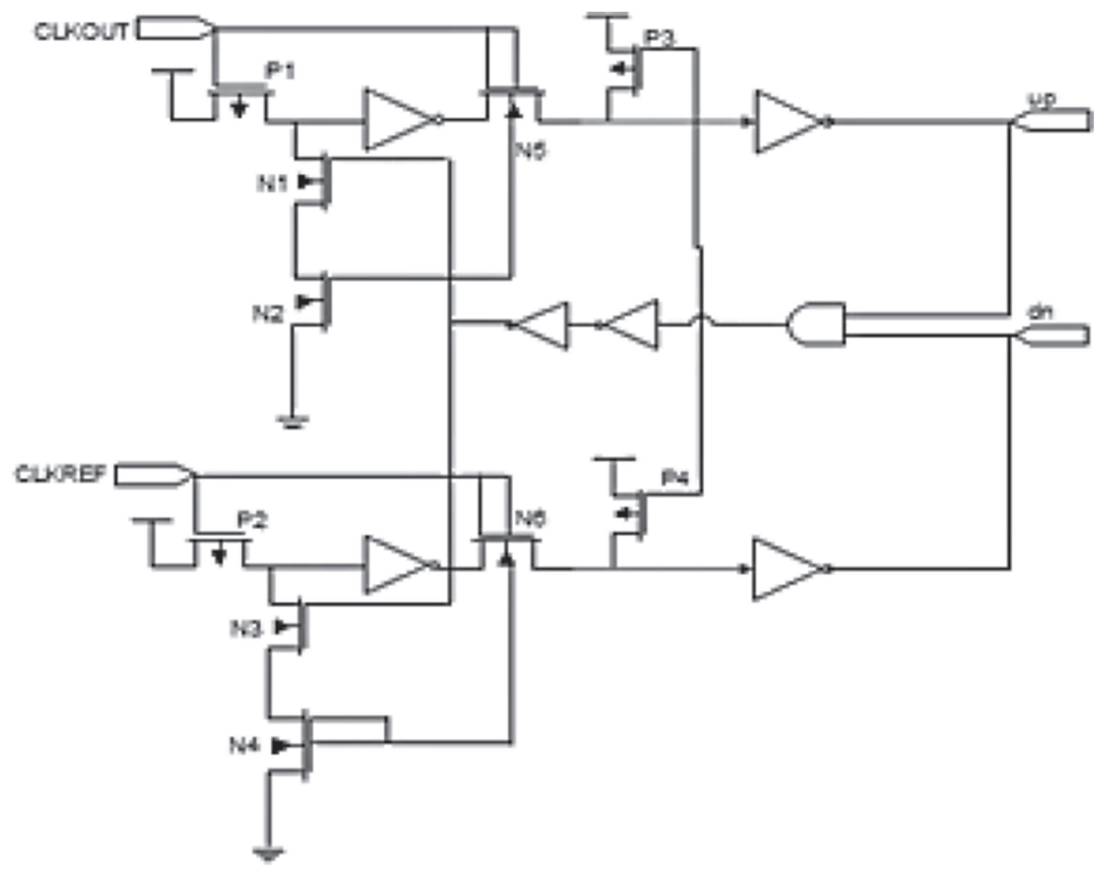

Figure 2. Proposed Pass transistor AND based PFD design.

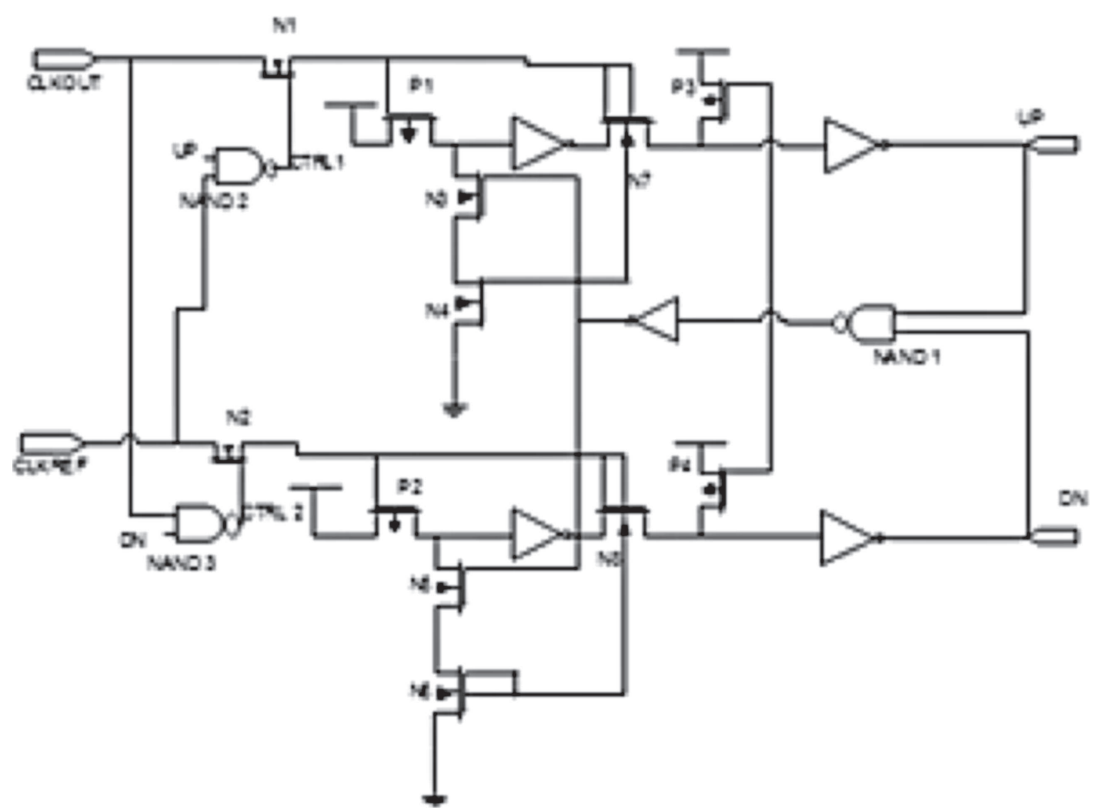

Figure 3. Proposed DIE Pass transistor PFD design. 


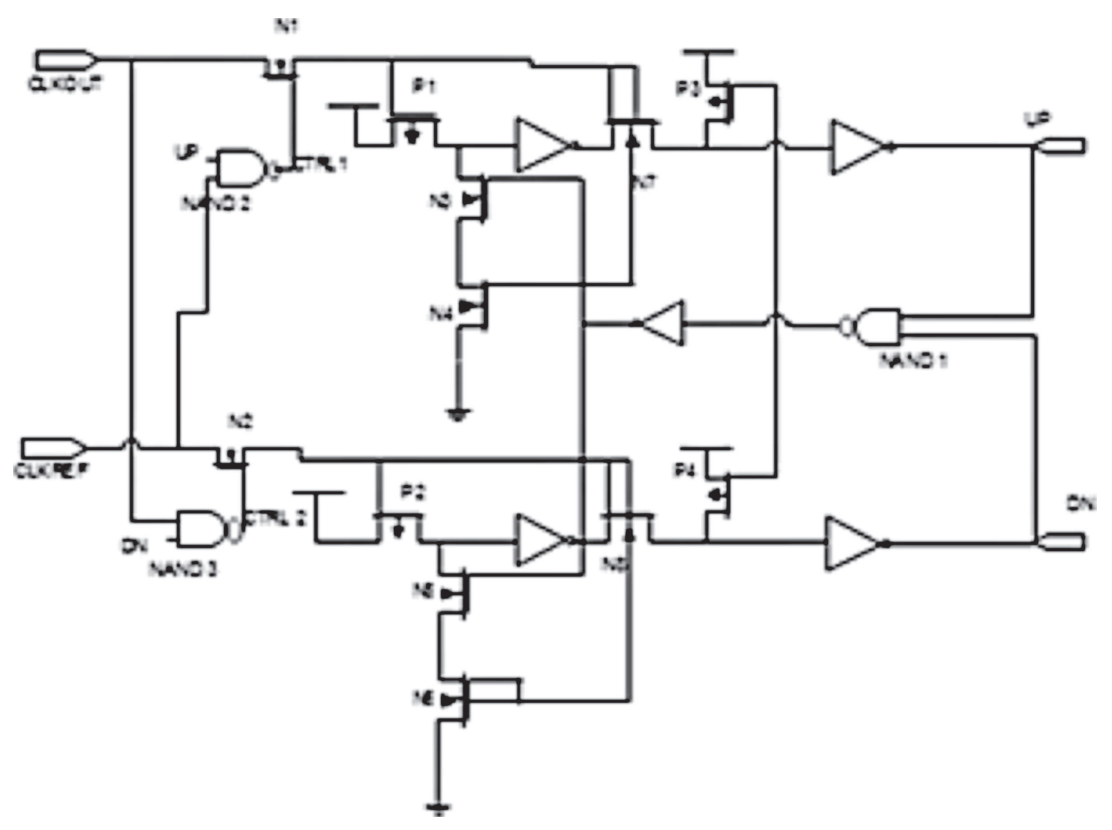

Figure 4. Proposed DIE Pass transistor NAND based PFD design.

\subsection{Proposed DIE pass transistor NAND based PFD design}

Two modified circuits which are designed above reduce the dead zone to a smaller extent only. Figure 4 illustrates the circuit consists of both DIE and Pass Transistor Logic design. In DIE Pass Transistor NAND based PFD, pass transistor logic is applied to all the NAND gates used (Markovic et al 2000).

Thus, the modified design has reduced power consumption and transistor count. By using the concept of DIE, the input delay occurs only during the reset phase. At the reset phase, N-MOS pass transistor is off; whereas the rising edge approaches the input is closed up. The input is propagated only after the reset phase (Zhang \& Syrzycki 2010). Therefore, the input delay is reached. Since this design consists of both DIE and Pass Transistor Logic architecture, the diminution of the dead zone to a greater extent when compared to all other existing patterns.

\subsection{Proposed design of improved CP}

The current mismatch in CP can even cause a spur in PLL. This improved CP circuit shows perfect current matching characteristics (Lee et al 2000). By extending the output impedance of conventional $\mathrm{CP}$, the sinking/sourcing current matching is improved. Figure 5 illustrates the improved single ended differential amplifier (Jae Hyung Noh \& Hang Geun Jeong 2007) which is placed in current-voltage feedback configuration and thus providing high output impedance. If UP and DN signals are high, then $\mathrm{I} 4=\mathrm{I} 3=\mathrm{I} 2$ and then if UP and DN signals are low, then I3 = I $2=\mathrm{I} 1$. From this, sink current I4 must be equal to source current I1 to check the current matching characteristics of CP. Figure 6 represents the proposed design of improved CP circuit, where differential amplifier with source follower and level shifter circuit is used. 


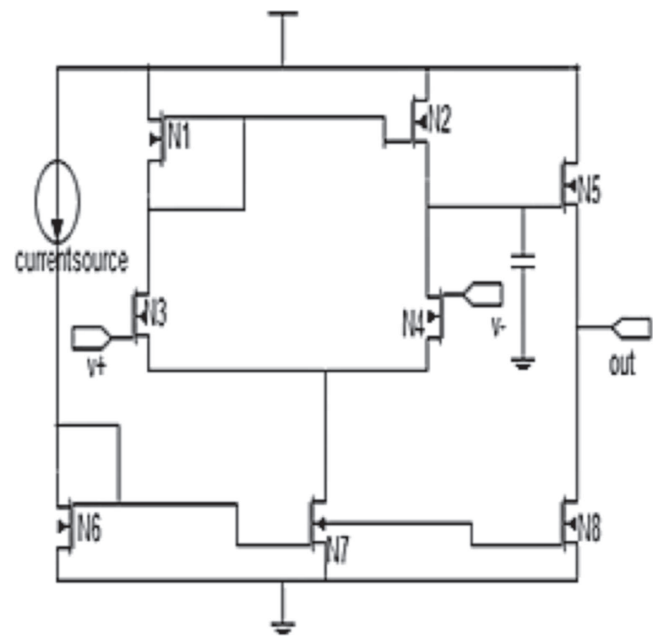

Figure 5. Improved single ended differential amplifier.

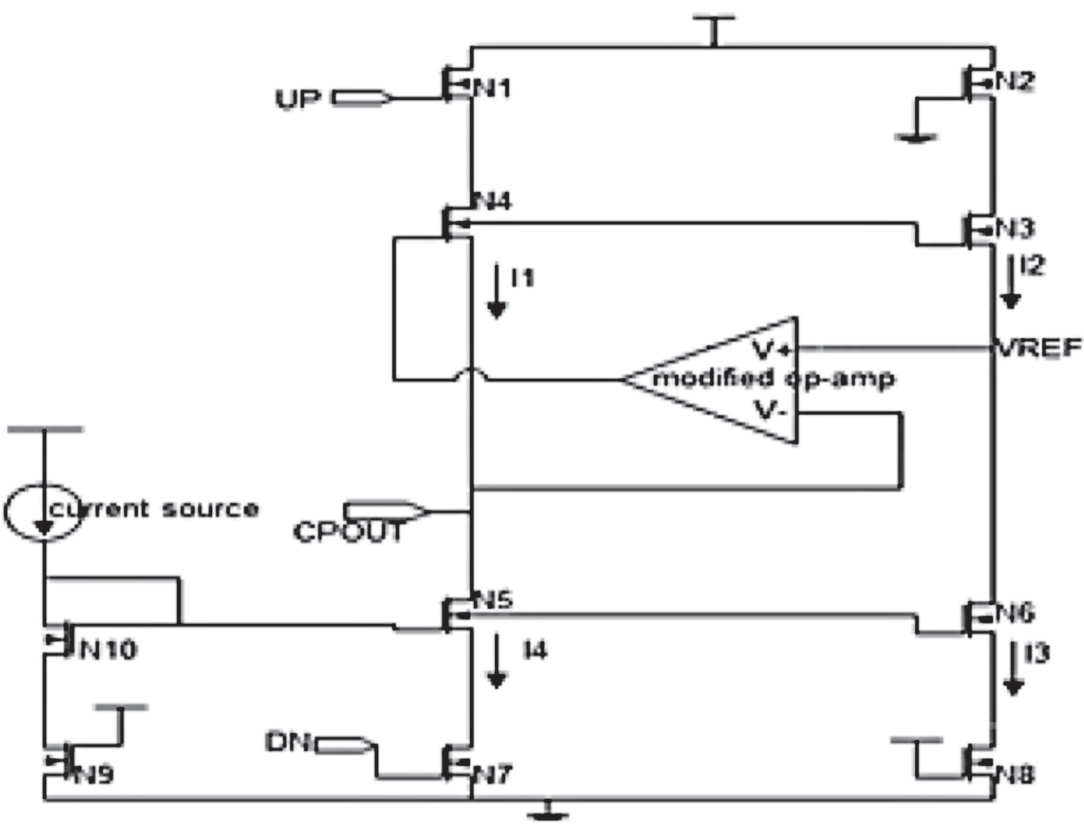

Figure 6. Proposed design of improved CP.

\section{Simulation results}

From the Literature survey, each and every circuit is designed, simulated using T-spice in IBM $130 \mathrm{~nm}$ technology. As per the comparative analysis (AnushKannan et al 2013a, b), pass transistor based PFD has the maximum dead zone. When the clock reference leads the clock out signal, 
Table 1. Comparison of existing and modified design of PFD.

\begin{tabular}{|c|c|c|c|c|c|}
\hline \multirow{2}{*}{$\begin{array}{l}\text { Module } \\
\text { technology }\end{array}$} & \multicolumn{2}{|c|}{ Dead zone (ns) } & \multicolumn{2}{|c|}{ Max power consumed $(\mu \mathrm{W})$} & \multirow[b]{2}{*}{ Transistor count } \\
\hline & $0.13 \mu \mathrm{m}$ & $0.18 \mu \mathrm{m}$ & $0.13 \mu \mathrm{m}$ & $0.18 \mu \mathrm{m}$ & \\
\hline Conventional PFD design & 0.5 & 0.7 & 131.64 & 146.83 & 68 \\
\hline $\begin{array}{l}\text { Pass transistor D Flip Flop } \\
\text { based PFD design }\end{array}$ & 1.0 & 1.3 & 107.71 & 112.62 & 24 \\
\hline $\begin{array}{l}\text { Proposed Pass transistor AND } \\
\text { based PFD design }\end{array}$ & 0.9 & 1.1 & 93.45 & 98.06 & 28 \\
\hline $\begin{array}{l}\text { Proposed DIE Pass transistor } \\
\text { PFD design }\end{array}$ & 0.8 & 0.9 & 87.59 & 90.34 & 32 \\
\hline $\begin{array}{l}\text { Proposed DIE Pass transistor NAND } \\
\text { based PFD design }\end{array}$ & 0.3 & 0.5 & 75.91 & 80.96 & 38 \\
\hline
\end{tabular}

their difference is appeared as output at UP signal. Similarly, when the clock out leads the clock reference signal and their difference will also be appeared as output at DN signal. Another condition is that when both clock out and clock reference signals are high at same time, there will be a smallest phase difference that appears as a dead zone. With this concept, the performance of the three modified PFD designs is observed. From the net list generated, power consumption of each circuit is evaluated. The simulation of the dead zone is done at $15 \mathrm{~ns}$ with both $1.3 \mathrm{~V}$ and $1.8 \mathrm{~V}$ power supply. Table 1 gives the comparative analysis of PFD circuits.

The DIE Pass transistor NAND based PFD circuit compares two clock signals and produces their phase error voltage as UP and DN signals. When one of the input clocks rise, the corresponding output also becomes high. This condition is maintained until the second clock signal goes high. This condition in turn resets the circuit and return the PFD back to original position.

Many existing designs of PFD are studied, designed, simulated and compared their results. From the comparison, it is found that Pass Transistor D Flip Flop Based PFD (Mansuri et al 2002) module has greater dead zone. So, three modified plans are proposed to overcome their dead zone problem. From the above three designs, it is found that DIE Pass transistor NAND based PFD design has minimum dead zone.

From tables 1 and 2 the observations are listed as follows

- By taking into account of the maximum power consumption, DIE Pass transistor NAND based PFD design shows minimum power consumed compared to other existing designs. The reduction in power consumption is mainly due to the replacement of CMOS NAND gate with Pass Transistor Logic based NAND gate.

Table 2. Performance measures of existing and modified design of PFD.

\begin{tabular}{lccc}
\hline Module & $\begin{array}{c}\text { Maximum operating } \\
\text { frequency (MHz) }\end{array}$ & Capture range (MHz) \\
\hline Supply voltage & $1.3 \mathrm{~V}$ & $1.8 \mathrm{~V}$ & $1.3 \mathrm{~V}$ \\
Conventional PFD design & 400 & 500 & $150-225$ \\
Pass transistor D Flip Flop based PFD design & 600 & 750 & $200-350$ \\
Proposed Pass transistor AND based PFD design & 750 & 800 & $200-400$ \\
Proposed DIE Pass transistor PFD design & 900 & 1000 & $200-500$ \\
Proposed DIE Pass transistor NAND based PFD design & 1000 & 1200 & $300-600$ \\
\hline
\end{tabular}


- While considering the transistor count, the DIE Pass transistor NAND based PFD design has a less transistor count than conventional PFD design.

- Results reveal that the circuits designed based on pass transistor logic reduce power and also area is minimized as the transistor count is decreased.

- With regard to the dead zone, the overall modifications done in Pass transistor D Flip Flop Based PFD design that has emerged as DIE Pass transistor NAND based PFD design which shows their minimum dead zone compared to other existing designs of the PFD.

- Maximum operating frequency and Capture range are observed for all the circuits designed with the supply voltage $1.3 \mathrm{~V}$ and $1.8 \mathrm{~V}$.

- While doing literature survey it is found that the existing Pass Transistor D Flip Flop based PFD design has the maximum dead zone. Reduction of dead zone is achieved with three modified designs keeping the above existing as reference. Conventional PFD design's performance is included to indicate the point that though it has a lower dead zone, power consumption is maximum. Moreover, there is only a smaller raise in dead zone value for the Proposed Pass transistor AND based PFD design and Proposed DIE Pass transistor PFD design compared to conventional PFD design but lower power consumption is achieved as there is a decrease in transistor count.

- Figure 7 illustrates dead zone output of DIE Pass transistor NAND based PFD design using $0.13 \mu \mathrm{m}$ TSPICE. The dead zone for this design is found to be $0.3 \mathrm{~ns}$. The maximum power consumed by this circuit is $75.91 \mu \mathrm{W}$.

- Figure 8 represents the comparison of dead zone for various PFD circuits.

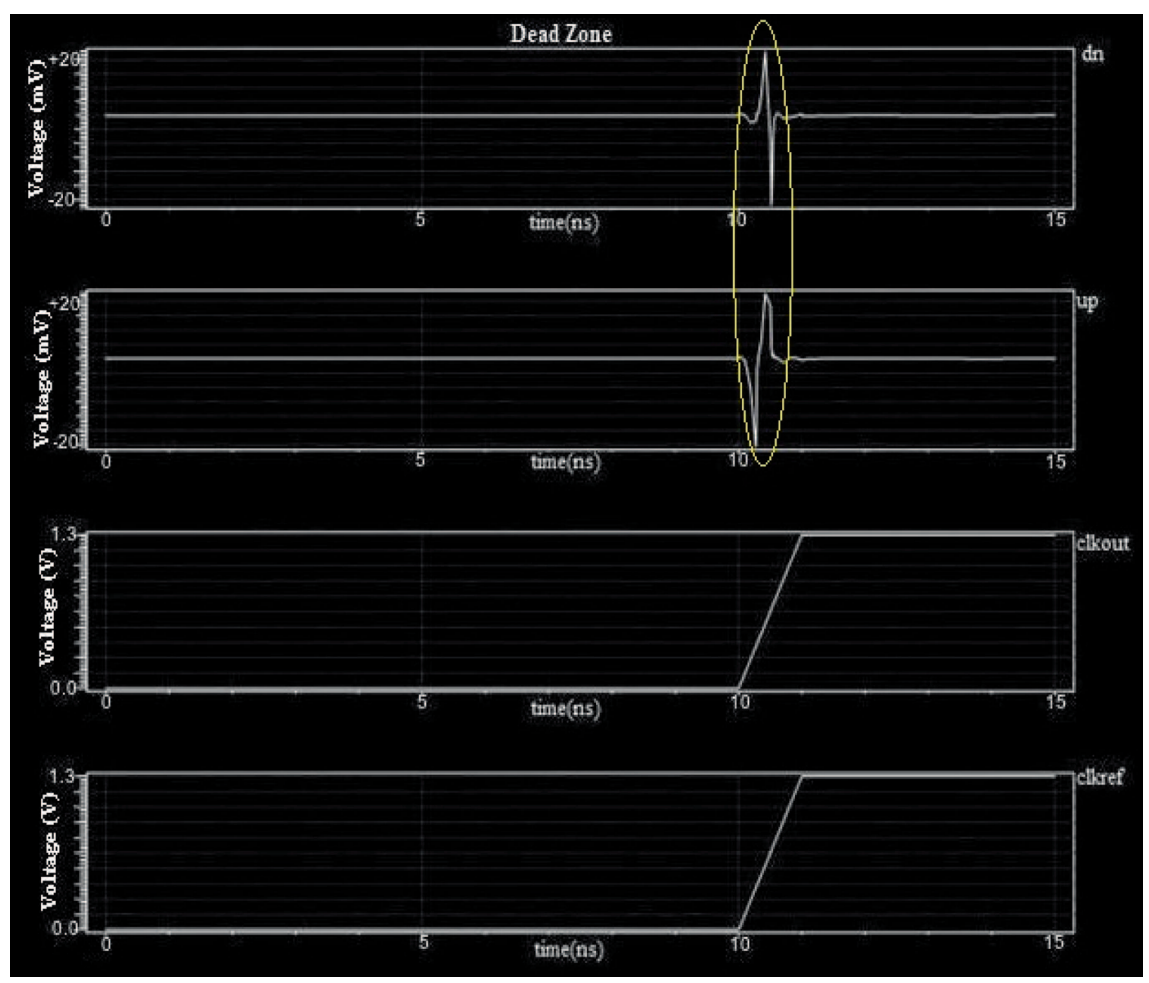

Figure 7. Dead zone result of DIE pass transistor NAND based PFD design. 


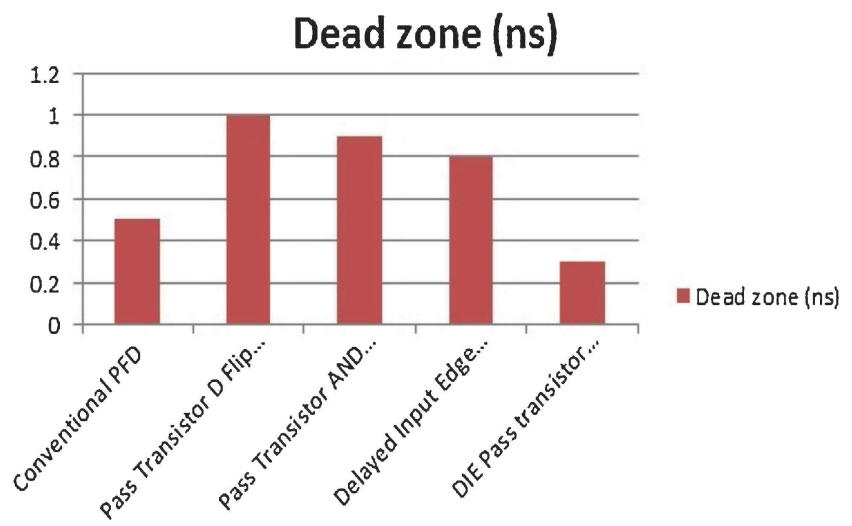

Figure 8. Comparison of dead zone.

Table 3. Comparison of existing and modified design of CP.

\begin{tabular}{lcc}
\hline Module & Offset delay $(\mathrm{ns})$ & Current mismatch $(\mu \mathrm{A})$ \\
\hline Conventional & 20.8 & 6 \\
Error amplifier & 21 & 0.28 \\
Improved & 6 & 0.1 \\
\hline
\end{tabular}

Table 4. Performance measures of improved design of CP.

\begin{tabular}{lc}
\hline Parameters & Values \\
\hline Supply voltage & $1.3 \mathrm{~V}$ \\
Operating frequency & $100 \mathrm{MHz}$ \\
Pull up \& down currents & $10 \mu \mathrm{A}$ \\
Rise \& fall time & $2 \mathrm{~ns}$ \\
Technology & $0.13 \mu \mathrm{m}$
\end{tabular}

From tables 3 and 4, the observations are listed in the following

- With regard to current mismatch, Improved CP design shows a minimum current mismatch when compared to conventional design. Implementation of op-amp in a feedback configuration shows the best result in reducing current mismatch.

- In Improved CP module, a single ended differential amplifier is added because ideal opamp can produce only zero output Impedance. A simple cascode circuit without an op-amp gives a small output impedance (Chiu 2013) which is given by the following equation:

$$
R_{o}=\left(g_{m 2} r_{o 1}+1\right) r_{o 2}+r_{o 1}
$$

- Whereas the op-amp with current-voltage feedback (active cascode gain) can result in high output impedance which is given by the following equation:

$$
R_{o}=\left[\left(A_{a}+1\right) g_{m 2} r_{o 1}+1\right] r_{o 2}+r_{o 1}
$$




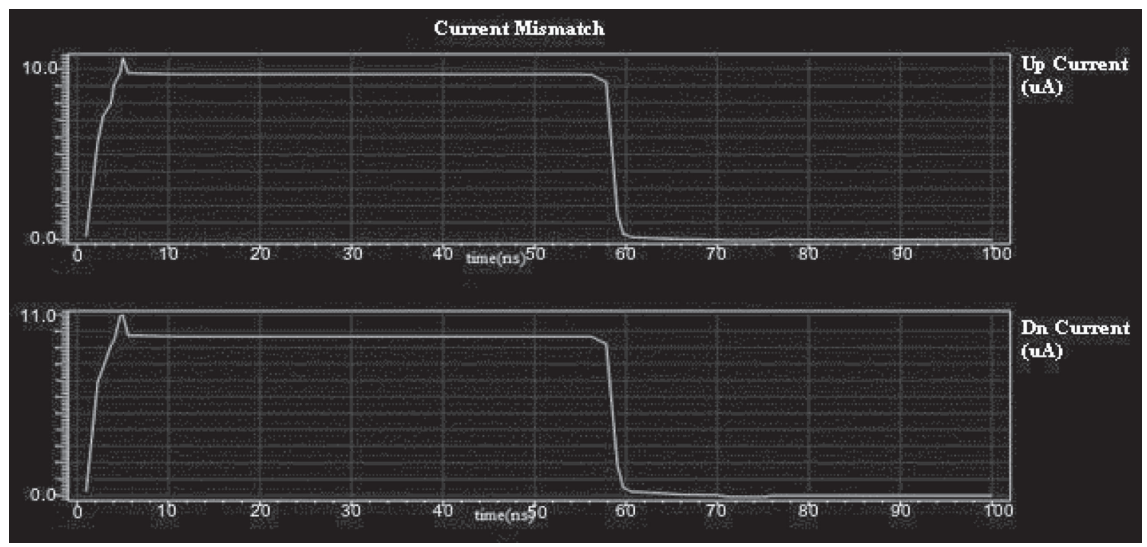

Figure 9. Current mismatch output of improved CP design.

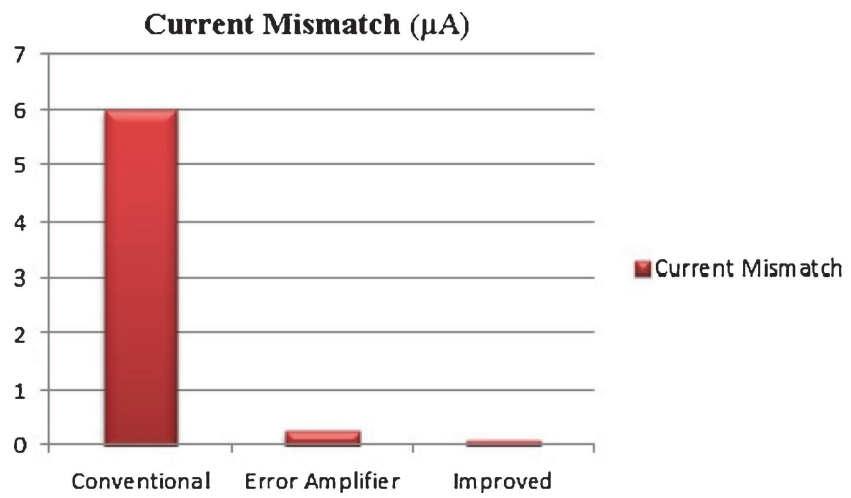

Figure 10. Comparison of current mismatch.

Table 5. Performance summary of PFD-CP module.

\begin{tabular}{lcc}
\hline Design specifications & \multicolumn{2}{c}{ Results } \\
\cline { 2 - 3 } Module name & DIE-pass transistor NAND based PFD design & Improved CP design \\
\hline Technology used & $0.13 \mu \mathrm{m}$ and $0.18 \mu \mathrm{m}$ & $0.13 \mu \mathrm{m}$ \\
Power supply & $1.3 \mathrm{~V}$ & $1.3 \mathrm{~V}$ \\
Transistor count & 38 & 18 \\
Maximum power consumption & $75.91 \mu \mathrm{W}$ & $280 \mathrm{nW}$ \\
Dead zone & $0.3 \mathrm{~ns}$ & - \\
Current mismatch & - & $0.1 \mu \mathrm{A}$ \\
\hline
\end{tabular}

- The extended output voltage ranges of the charge-pump, where the amplifier is placed in current voltage feedback configuration reduces the mismatch between the Up current and the Down current with decreased offset delay and thereby increasing output impedance.

- Overall performance measures are observed with the proposed design using $0.13 \mu \mathrm{m}$ TSPICE

- Figure 9 illustrates the current mismatch result of improved $\mathrm{CP}$ design. The current mismatch is found to be $0.1 \mu \mathrm{A}$ and the time delay is $6 \mathrm{~ns}$. 


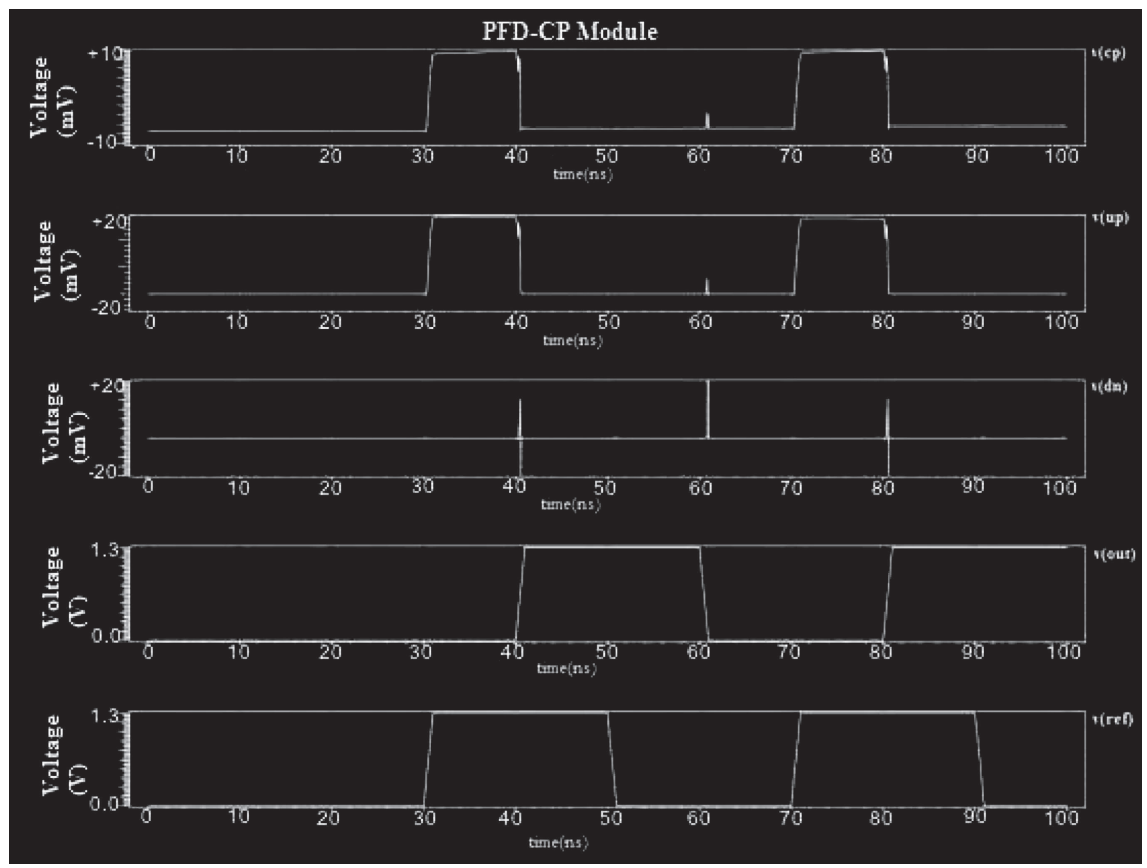

Figure 11. Simulation output for overall module.

- Figure 10 represents the values of current mismatch observed for various existing $\mathrm{CP}$ circuits with the proposed design.

- The CP simulation is done under $1.3 \mathrm{~V}$ supply (table 5).

From this by combining Modified PFD module which has reduced dead zone and Improved $\mathrm{CP}$ design that has reduced mismatch, the overall module is designed. This circuit is simulated under $1.3 \mathrm{~V}$ power supply which is illustrated in figure 11 . Performance of overall module is summarized in table 3 .

\section{Conclusion}

In this paper, three PFD designed circuits reduce the dead zone and an improved CP design reduces the current mismatch. All the modified circuits are designed, simulated and their outputs are observed. From the comparative analysis, it is found that DIE Pass transistor NAND based PFD design has minimum dead zone of $0.3 \mathrm{~ns}$ and the power consumed is about $75.91 \mu \mathrm{W}$ and also improved CP design has a minimum current mismatch of $0.1 \mu \mathrm{A}$. Thus the DIE Pass transistor NAND based PFD design with minimum dead zone and improved CP design with reduced current mismatch interfaced to obtain modified PFD-CP architecture for a PLL implemented using IBM $0.13 \mu \mathrm{m}$ technology under a supply voltage of $1.3 \mathrm{~V}$.

\section{References}

AnushKannan N K, Dharani V A, Divya G, Esack N, Gokulraj M and Mangalam H 2013a Comparison and analysis of various PFD architecture for a phase locked loop design. In: Computational 
Intelligence and Computing Research (ICCIC), 2013 IEEE international conference on IEEE, pp. 105108

AnushKannan N K, Dharani V A, Divya G, Esack N, Gokulraj M and Mangalam H 2013b Design of various PFD and charge-pump architectures for a PLL-a survey. CiiT Int. J. Digital Signal Process. 5: 284-287

Bai Na, Ji Xincun, Guan Weiping and Lin Zhiting 2014 An improved charge pump with suppressed charge sharing effect. TELKOMNIKA Indonesian J. Electrical Eng. 12: 1245-1249

Best R E 2003 Introduction to PLLs, Phase-locked loops: Design, simulation and applications, Fifth Edition, Newyork, USA, Buch McGraw-Hill, chapter 1

Cheng Zhang and Marek Syrzycki 2010 Modifications of a dynamic-logic phase frequency detector for extended detection range. In: Circuits and systems (MWSCAS), 53rd IEEE international midwest symposium, IEEE pp 105-108

Dong-Keon Lee, Jeong-Kwang Lee and Hang-Geun Jeong 2010 A dual compensated charge pump with reduced current mismatch. In: Proceedings of the 4th WSEAS international conference on circuits, systems, signal and telecommunications, World Scientific and Engineering Academy and Society (WSEAS), pp. 109-112

Jae Hyung Noh and Hang Geun Jeong 2007 Charge-pump with a regulated cascode circuit for reducing current mismatch in PLLs. World Acad. Sci. Eng. Technol., Int. J. Electrical, Robotics, Electron. Commun. Eng. 1: 1001-1003

Jae-Shin Lee, Min-Sun Keel, Shin-I1 Lim and Suki Kim 2000 Charge pump with perfect current matching characteristics in phase-locked loops. Electron. Lett. 36: 1907-1908

Jayashree Nidagundi, Harish Desai, Shruti A and Gopal Manik 2013 Design and Implementation of Low Power Phase Frequency Detector (PFD) for PLL. Int. J. Sci. Eng. Technol. 2: 160-163

Khare K, Khare N, Deshpande P and Kulhade V 2008 Phase frequency detector of the delay locked loop at high frequency. In: Semiconductor electronics IEEE international conference (ICSE2008), pp 113-116

Kristen Elserougi, Ranil Fernando and Luca Wei 2006 Phase locked loop design, PhD dissertation, School of Engineering, Santa Clara University, Santa Clara, California

Kun-Seok Lee, Byeong-Ha Park, Han-il Lee and Min Jong Yoh 2003 Phase frequency detectors for fast frequency acquisition in Zero-dead-zone CPPLLs for mobile communication systems, Solid-state circuits conference, 2003 ESSCIRC'03. In: Proceedings of the 29th European IEEE, pp. 525-528

Mano M, Selva Priya G and RekhaSwathi Sri K 2013 Design and implementation of modified charge pump for phase locked loop. Int. J. Emerging Technol. Adv. Eng. 3: 558-562

Marković D, Nikolić B and Oklobdžija V G 2000 A general method in synthesis of pass-transistor circuits. Microelectron. J. 31: 991-998

Mhd Zaher Al Sabbagh 20070.18 um phase/frequency detectors and charge pump design for digital video Broadcasting for handheld's phase-locked-loop systems MS dissertation, Graduate school of the Ohio State University, Ohio

Mozhgan Mansuri, Dean Liu and Chih-Kong Ken Yang 2002 Fast frequency acquisition phase-frequency detectors for GSamples/s phase-locked loops. IEEE J. Solid-State Circuits 37: 1331-1334

NehaPathak and Ravi Mohan 2014 Performance analysis and implementation of CMOS current starved voltage controlled oscillator for phase locked loop. Int. J. Emerging Technol. Adv. Eng. 4: 365-369

Talwekar R H and Limaye S S 2010 Design of high gain, high bandwidth Op-Amp for reduction of mismatch currents in charge pump PLL in $180 \mathrm{~nm}$ CMOS technology. World Acad. Sci. Eng. Technol. 72: 590-593

Wen-Ching Chang, Chun-Hung Lien and Yu-Chung Wei 2002 A fully integrated CMOS PLL for frequency synthesizer using Gm-C filter. In: Proceedings WSEAS international conference, World Scientific and Engineering Academy and Society (WSEAS), pp 451-279

Whitaker S 1983 Pass-transistor networks optimize n-MOS logic. Electronics Letters, Penton Publishing 56: $144-148$

Xintian Shi 2008 Design of low phase noise low power CMOS phase locked loops. PhD dissertation, Faculty of Sciences, University of Neuchatel, Switzerland

Yun Chiu 2013 On the operation of CMOS active-cascode gain stage. J. Comput. Commun. 1: 18 